\title{
Downregulation of the long non-coding RNA ZFAS1 is associated with cell proliferation, migration and invasion in breast cancer
}

\author{
SHULIN FAN ${ }^{1}$, CHUNNI FAN $^{1}$, NING LIU $^{1}$, KEKE HUANG $^{2}$, XUEDONG FANG $^{3}$ and KEREN WANG ${ }^{1}$ \\ ${ }^{1}$ Department of Breast Surgery, China-Japan Union Hospital of Jilin University, Changchun, Jilin 130033; \\ ${ }^{2}$ State Key Laboratory of Inorganic Synthesis and Preparative Chemistry, College of Chemistry, \\ Jilin University, Changchun, Jilin 130012; ${ }^{3}$ Department of Gastrointestinal Surgery, \\ China-Japan Union Hospital of Jilin University, Changchun, Jilin 130033, P.R. China
}

Received November 1, 2017; Accepted March 1, 2018

DOI: $10.3892 / \mathrm{mmr} .2018 .8707$

\begin{abstract}
Long non-coding RNAs (lncRNAs) are non-coding RNAs that are $>200$ nucleotides in length. Recent studies have identified a number of lncRNAs with critical roles in various biological processes including tumorigenesis. Zinc finger antisense 1 (ZFAS1) is a lncRNA that has recently been reported to be involved in the progression of several human cancers. However, the biological function of ZFAS1 in breast cancer remains to be elucidated. In order to determine the effect of ZFAS1 in breast cancer cells, reverse transcription-quantitative polymerase chain reaction (RT-qPCR) was performed to measure ZFAS1 expression in cells from breast cancer cell lines. In addition, gain-of-function experiments were performed in vitro to investigate the biological role of ZFAS1. The results revealed that ZFAS1 expression was significantly downregulated in breast cancer cell lines when compared with the levels in controls. In vitro experiments also demonstrated that ZFAS1 overexpression significantly suppressed cell proliferation by causing cell cycle arrest and inducing apoptosis in breast cancer cells. Further functional assays indicated that ZFAS1 overexpression inhibited cell migration and invasion by regulating epithelial-mesenchymal transition. These findings indicated that the lncRNA ZFAS1 may be a tumor suppressor in breast cancer, and thus, may serve as a potential therapeutic target for patients with breast cancer.
\end{abstract}

Correspondence to: Professor Xuedong Fang, Department of Gastrointestinal Surgery, China-Japan Union Hospital of Jilin University, 126 Xiantai Street, Changchun, Jilin 130033, P.R. China E-mail: xuedongfang8866@163.com

Professor Keren Wang, Department of Breast Surgery, China-Japan Union Hospital of Jilin University, 126 Xiantai Street, Changchun, Jilin 130033, P.R. China

E-mail: kerenwang88@163.com

Key words: zinc finger antisense 1, long non-coding RNA, breast cancer, cell proliferation, epithelial-mesenchymal transition

\section{Introduction}

Breast cancer is the most commonly diagnosed cancer and principal cause of death among females worldwide (1). Despite enormous efforts towards characterizing the underlying molecular mechanisms of breast cancer, the potential mechanisms that regulate breast cancer progression remain largely elusive and poorly defined. Clinically, although advances have been made in the treatment of breast cancer over the last decade, including the development of personalized treatment based on four molecular types of breast cancer (luminal A, luminal B, HER2-positive and triple-negative breast cancer), the effective control of recurrence and metastasis remains the biggest obstacle. Therefore, a better understanding of tumorigenesis and the identification of novel therapeutic targets are urgently needed for the treatment of patients with breast cancer.

Long non-coding RNAs (lncRNAs) are a class of RNA transcripts over 200 nucleotides in length that lack protein-coding capacity $(2,3)$. Over the last few decades, accumulating evidence has suggested the importance of lncRNAs in the regulation of biological processes, such as cell differentiation, development, and apoptosis (4). An increasing number of lncRNAs have been shown to participate in many human diseases, including malignancy $(5,6)$. Aberrant lncRNA expression has been reported in different types of cancers, exhibiting both oncogenic and cancer-suppressive roles (7-9). These findings suggest that the dysregulation of IncRNAs may be an important cause of tumorigenesis and accelerate tumor progression.

ZFAS1 (zinc finger antisense 1) is a newly identified lncRNA that regulates alveolar development and epithelial cell differentiation in the mouse mammary gland (10). Recently, it was reported that ZFAS1 is aberrantly expressed in cancer and is involved in the progression of different types of tumors. It is upregulated in gastric, colonic, glioma, and ovarian cancer and function as a potential oncogene by promoting cell proliferation and metastasis (11-14). In contrast, Askarian-Amiri et al (10) found that ZFAS1 is downregulated in invasive ductal breast carcinoma compared to levels in normal breast tissue, a finding which leads the authors to speculate that ZFAS1 serves as a tumor suppressor. However, the biological role of ZFAS1 in breast cancer and its underlying molecular mechanism are unclear and therefore served as the focus of the present study. 
In this study, we found that ZFAS1 expression was downregulated in different human breast cancer cell lines, and additional experiments further demonstrated that overexpression of ZFAS1 inhibited breast cancer cell proliferation, migration, and invasion in vitro. Mechanistically, we verified that ZFAS1 inhibited breast cancer cell migration and invasion by regulating the epithelial-mesenchymal transition (EMT) process. Therefore, our results suggest that ZFAS1 acts as a tumor suppressor in breast cancer and may be an effective therapeutic target for patients with breast cancer.

\section{Materials and methods}

Cell culture. Four human breast cancer cell lines (MDA-MB-231, MCF-7, T-47D, and SK-BR-3) and a normal breast epithelial cell line (MCF-10A) were obtained from the American Type Culture Collection (ATCC; Rockefeller, MD, USA). Cells were cultured in RPMI-1640, Dulbecco's modified Eagle's medium (DMEM), or DMEM/F12 medium (HyClone; GE Healthcare Life Sciences, Logan, UT, USA), containing 10\% fetal bovine serum (FBS; HyClone; GE Healthcare Life Sciences), and were incubated at $37^{\circ} \mathrm{C}$ in an incubator containing $5 \% \mathrm{CO}_{2}$.

RNA extraction and reverse transcription-quantitative polymerase chain reaction $(R T-q P C R)$. Total RNA was isolated from cultured cells using TRIzol reagent (Invitrogen; Thermo Fisher Scientific, Inc., Waltham, MA, USA) according to the manufacturer's instructions. RNA concentration and purity were determined using a NanoDrop spectrophotometer (Thermo Fisher Scientific, Inc.). Total RNA (500 ng) was reverse transcribed into cDNA using PrimeScript RT Master Mix (Takara Biotechnology Co., Ltd., Dalian, China) following the manufacturer's protocol. RT-qPCR was performed on an Eppendorf Real-time PCR System (Eppendorf, Hamburg, Germany) using the SYBR PrimeScript RT-PCR kit (Takara Biotechnology Co., Ltd.) with specific primers. GAPDH was used as an internal control. The primers used for RT-qPCR were as follows: ZFAS1, forward: 5'-GAGGTTCAGGAAGCC ATTCGTTCT-3' and reverse: 5'-CCAGTGGTGACTCCC TCTTCCAAA-3'; GAPDH, forward: 5'-GAAGGCTGGGGC TCATTTGCAGGG-3' and reverse: 5'-GGTGCAGGAGGC ATTGCTGATGAT-3'. PCR reaction mixtures $(25 \mu \mathrm{l})$ were prepared, including $12.5 \mu \mathrm{l} \mathrm{SYBR-Green} \mathrm{permix,} 2 \mu \mathrm{l} \mathrm{cDNA}$, $0.75 \mu \mathrm{l}$ of each primer and $9 \mu \mathrm{l}$ double-distilled water to a final volume of $25 \mu \mathrm{l}$. The thermocycling conditions were as follows: $95^{\circ} \mathrm{C}$ for $30 \mathrm{sec}$, followed by 40 cycles of denaturation at $95^{\circ} \mathrm{C}$ for $5 \mathrm{sec}$, annealing at $60^{\circ} \mathrm{C}$ for $30 \mathrm{sec}$, and extension at $72^{\circ} \mathrm{C}$ for $30 \mathrm{sec}$. Relative expression fold-changes were calculated using the $2^{-\Delta \Delta \mathrm{Cq}}$ method. Each experiment was performed in triplicate.

Plasmid construction and cell transfection. Full-length human ZFAS1 was synthesized and subcloned into a pcDNA3.1 vector (Invitrogen; Thermo Fisher Scientific, Inc.), creating pcDNA-ZFAS1 for ZFAS1 overexpression analysis. An empty pcDNA3.1 vector was used as a control. To establish cell lines with stable overexpression of ZFAS1, cells were grown on 6-well plates to $60-70 \%$ confluence and transfected with $5 \mu \mathrm{g}$ plasmid using Lipofectamine 2000 reagent (Invitrogen; Thermo Fisher Scientific, Inc.) and Opti-MEM (Gibco;
Thermo Fisher Scientific, Inc.) according to the manufacturer's protocol. Cells were incubated at $37^{\circ} \mathrm{C}$ in a $\mathrm{CO}_{2}$ incubator for $48 \mathrm{~h}$ prior to evaluation for ZFAS1 expression and harvesting for further analysis. The medium was replaced after $6 \mathrm{~h}$ post-transfection.

Cell proliferation assay. Cell proliferation was measured using the Cell Counting Kit-8 (CCK-8; Beyotime Institute of Biotechnology, Shanghai, China), according to the manufacturer's protocol. Cells were seeded in 96-well plates $\left(5 \times 10^{3}\right.$ cells/well) and grown in $100 \mu \mathrm{l}$ culture medium. Cell viability was examined every $24 \mathrm{~h}$ for a total of five days. At each time point, $10 \mu \mathrm{l}$ CCK-8 reagents was added to each well, followed by incubation for $2 \mathrm{~h}$ at $37^{\circ} \mathrm{C}$. The absorbance of each well was then measured on a spectrophotometer (BioTek, Winooski, VT, USA) at $450 \mathrm{~nm}$. The assay was performed in three replicate wells, and three parallel experiments were performed for each sample.

Colony formation assay. Transfected cells were harvested after trypsinization and seeded in 6 -well plates $\left(8 \times 10^{2}\right.$ cells/well). The culture medium was refreshed every three days. Once colony formation was visible, the cells were rinsed twice with phosphate-buffered saline (PBS) followed by fixation with $4 \%$ paraformaldehyde and staining with Giemsa for $20 \mathrm{~min}$. The number of colonies was counted under a light microscope (Nikon, Tokyo, Japan), and each colony contained at least 50 cells.

5-Ethynyl-2-deoxyuridine (EdU) assay. To intuitively observe cell proliferation, an EdU incorporation assay was performed using an EdU Apollo in vitro Imaging kit (Ribobio, Guangzhou, China) according to the manufacturer's instructions. The transfected cells were cultured in 96-well plates at a density of $6 \times 10^{3}$ cells per well and grown to $60-80 \%$ confluence. Next, EdU labeling medium $(50 \mu \mathrm{M})$ was added to each well, followed by incubation for $2 \mathrm{~h}$ at $37^{\circ} \mathrm{C}$. Subsequently, cells were stained with anti-EdU working solution and Hoechst 33342 was used to label cell nuclei. EdU-positive cells were visualized by a fluorescent microscope (Olympus, Tokyo, Japan) and the percentage of EdU-positive cells was calculated.

Flow cytometry analysis. For the cell cycle assay, cells were harvested and washed three times with ice cold PBS. Subsequently, cells were fixed in $70 \%$ ethanol at $4^{\circ} \mathrm{C}$ for $24 \mathrm{~h}$ and then stained with propidium iodide (PI) using the Cell Cycle Analysis kit (Beyotime Institute of Biotechnology). The percentage of cells in each phase of the cell cycle was calculated and compared using Modfit LT software 3.1 (Verity Software House, Groton, MA, USA). To measure the cell apoptosis rate, transfected cells were harvested and washed using PBS. An Annexin V-FITC Apoptosis Detection kit (Beyotime Institute of Biotechnology) was used to detect cell apoptosis. According to the manufacturer's instructions, cells were stained with fluorescein isothiocyanate (FITC)-Annexin V and PI, and were incubated in the dark for $20 \mathrm{~min}$ at room temperature. Cell apoptosis was analyzed using a flow cytometer (BD Biosciences, Franklin Lakes, NJ, USA).

Cell invasion and migration assay. Migration and invasion assays were performed using a 24-well Transwell plate 
A

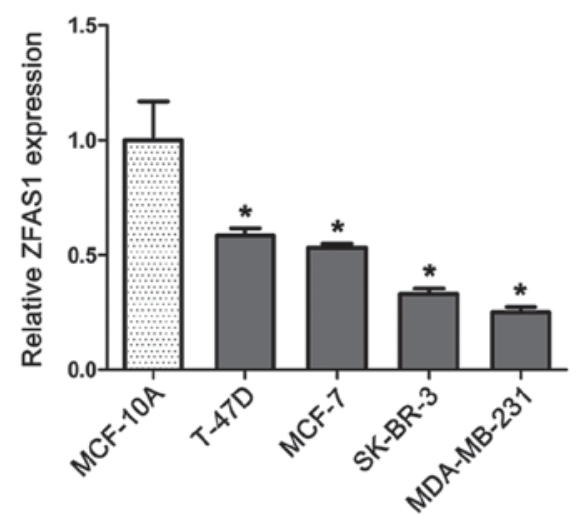

B

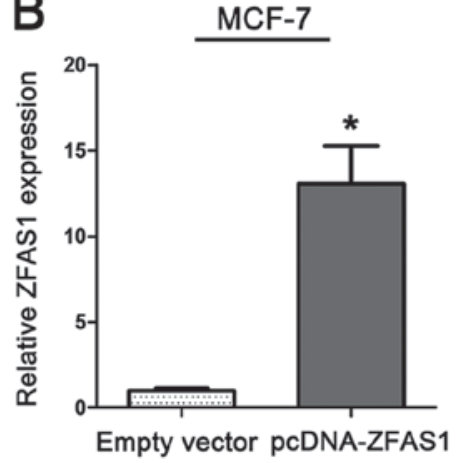

MDA-MB-231

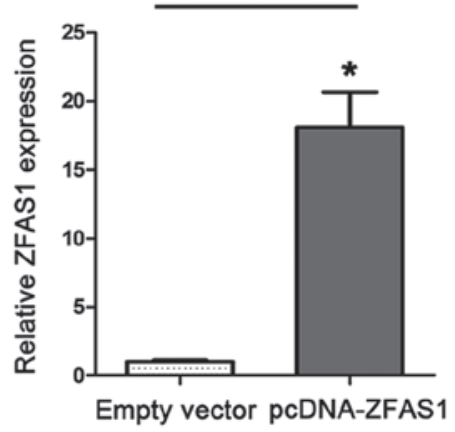

Figure 1. Relative ZFAS1 expression levels in breast cancer cell lines. Relative ZFAS1 expression levels were detected using reverse transcription-quantitative polymerase china reaction. (A) ZFAS1 was downregulated in cells from 4 breast cancer cell lines when compared with the levels in normal cells from the breast epithelial cell line, MCF-10A (control). (B) Transfection with pcDNA-ZFAS1 significantly upregulated ZFAS1 expression in the MCF-7 and MDA-MB-231 breast cancer cells when compared with the empty vector control. All experiments were performed in triplicate. Data are presented as the mean \pm standard deviation. ${ }^{*} \mathrm{P}<0.05$ vs. associated control group. ZFAS1, zinc finger antisense 1.

(8 $\mu \mathrm{m}$ pore size; Corning Incorporated, Corning, NY, USA). For migration assays, the upper chamber of the Transwell inserts was filled with $5 \times 10^{4}$ cells in $200 \mu 1$ serum-free DMEM. For invasion assays, cells $\left(5 \times 10^{4}\right)$ were seeded in the upper chamber coated with Matrigel (Corning Incorporated). Inserts were added to the bottom chamber wells containing $600 \mu \mathrm{l}$ of DMEM with $30 \%$ FBS. Next, the Transwell chambers were incubated for $24 \mathrm{~h}$ at $37^{\circ} \mathrm{C}$; then, the upper surface of the chamber was wiped with cotton swabs, and the cells on the filter surface were fixed with $4 \%$ paraformaldehyde and stained with Giemsa. The number of migratory and invasive cells was counted and photographed using a digital microscope (Nikon).

Western blotting. Cells were harvested after $48 \mathrm{~h}$ post-transfection and lysed with RIPA buffer (Beyotime Institute of Biotechnology) for protein extraction. Total protein concentration was quantified by the BCA Protein Assay kit (Beyotime Institute of Biotechnology). Equal amounts of protein $(20 \mu \mathrm{g})$ for each sample were loaded and separated by $10 \%$ sodium dodecyl sulfate polyacrylamide gel electrophoresis (SDS-PAGE) gels and then transferred to polyvinylidene difluoride (PVDF) membranes. The membranes were blocked with 5\% skim milk in Tris-buffered saline and Tween-20 at room temperature for $1 \mathrm{~h}$. The following primary antibodies were added and the membranes were incubated with gentle shaking at $4^{\circ} \mathrm{C}$ overnight: anti-E-cadherin (dilution, 1:200; cat. no. sc-21791; Santa Cruz Biotechnology, Inc., Santa Cruz, CA, USA), anti-vimentin (dilution, 1:200; cat. no. sc-6260; Santa Cruz Biotechnology, Inc.), and anti- $\beta$-actin (dilution, 1:1,000; cat. no. 4970; Cell Signaling Technology, Inc., Danvers, MA, USA). Then, the membranes were incubated with horseradish peroxidase (HRP)-conjugated secondary antibodies (dilution, 1:200; cat. no. sc-2380; Santa Cruz Biotechnology, Inc.) at room temperature for $1 \mathrm{~h}$. Finally, protein bands were visualized using an enhanced chemiluminescence (ECL) detection system (Thermo Fisher Scientific, Inc.).

Statistical analysis. All statistical analyses were performed using SPSS 21.0 (IBM Corp., Armonk, NY, USA). Data from the different groups were compared and analyzed using two-way analysis of variance and Bonferroni's post hoc test. Data are presented as the mean \pm standard deviation. Two-sided $\mathrm{P}$-values were calculated and $\mathrm{P}<0.05$ was considered to indicate a statistically significant difference.

\section{Results}

ZFAS1 is downregulated in breast cancer cell lines. To investigate the biological function of ZFAS1 in breast cancer tumorigenesis, we determined ZFAS1 expression levels in four breast cancer cell lines (MCF-7, T-47D, MDA-MB-231, and SK-BR-3) and a normal breast epithelial cell line (MCF-10A). We found that expression of ZFAS1 was significantly downregulated in cells from breast cancer cell lines compared to levels in cells from the normal breast epithelial cell line MCF-10A (Fig. 1A).

Overexpression of ZFAS1 inhibits breast cancer cell proliferation by causing cell cycle arrest. Furthermore, we performed CCK-8, colony formation, cell cycle, and EdU assays to evaluate the effect of ZFAS1 on cell proliferation in two breast cancer cell lines, MCF-7 and MDA-MB-231. Cells from the MCF-7 and MDA-MB-231 cell lines were transfected with pCDNA-ZFAS1 to stably overexpress ZFAS1. ZFAS1 overexpression in these two cell lines was confirmed by RT-qPCR (Fig. 1B). As shown in Fig. 2A, ZFAS1 overexpression significantly inhibited cell viability both in MCF-7 and MDA-MB-231 cells compared to that found in control cells. In addition, ZFAS1 overexpression in MCF-7 and MDA-MB-231 cells resulted in significantly decreased clonogenic survival (Fig. 2B). This finding was also confirmed by EdU/Hoechst immunostaining. As shown in Fig. $2 \mathrm{C}$, the percentage of EdU-positive cells was significantly decreased after ZFAS1 overexpression.

Overexpression of ZFAS1 promotes cell cycle arrest and induces apoptosis in breast cancer cells. To determine the potential role of ZFAS1 in breast cancer cell proliferation, we used flow cytometry to examine the function of ZFAS1 in breast cancer cell cycle progression and apoptosis. As shown in 
A

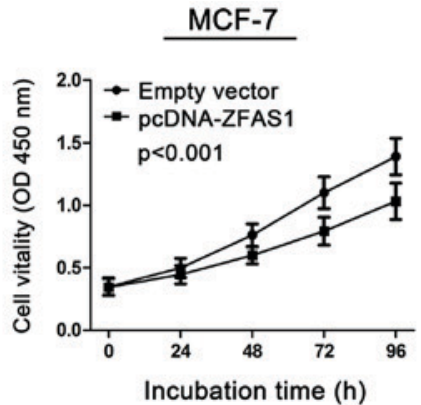

MDA-MB-231

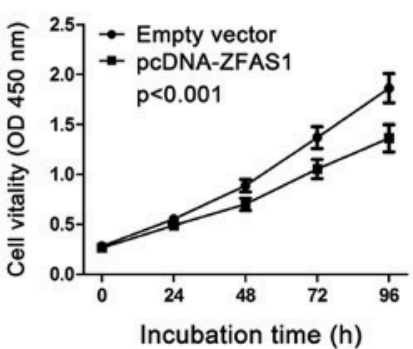

B

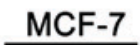

Empty vector pcDNA-ZFAS1
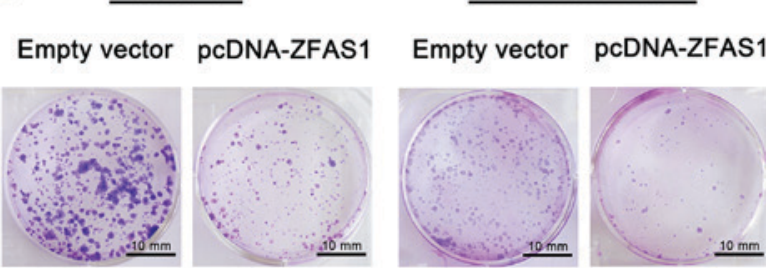

MDA-MB-231

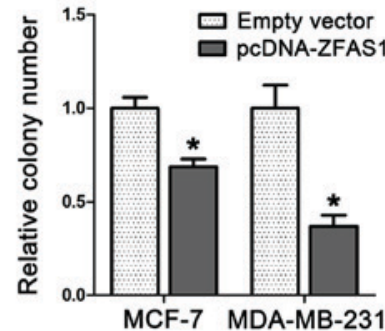

C

MCF-7
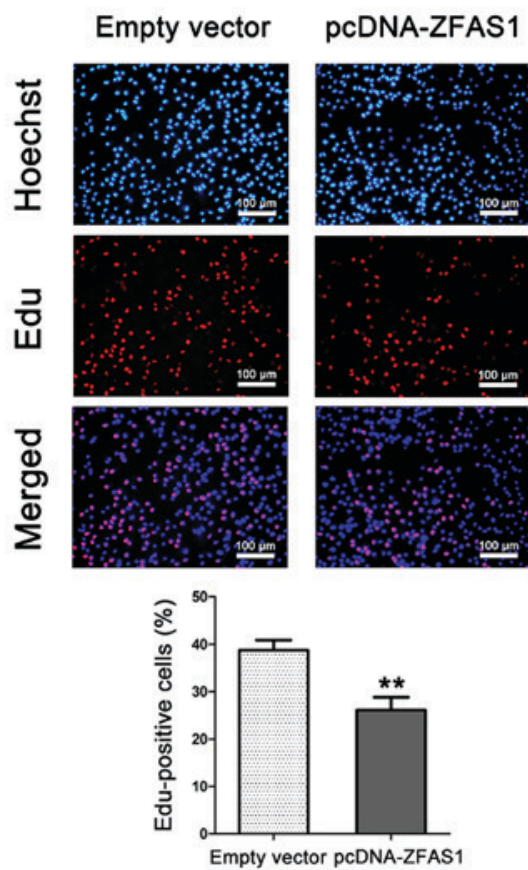

MDA-MB-231
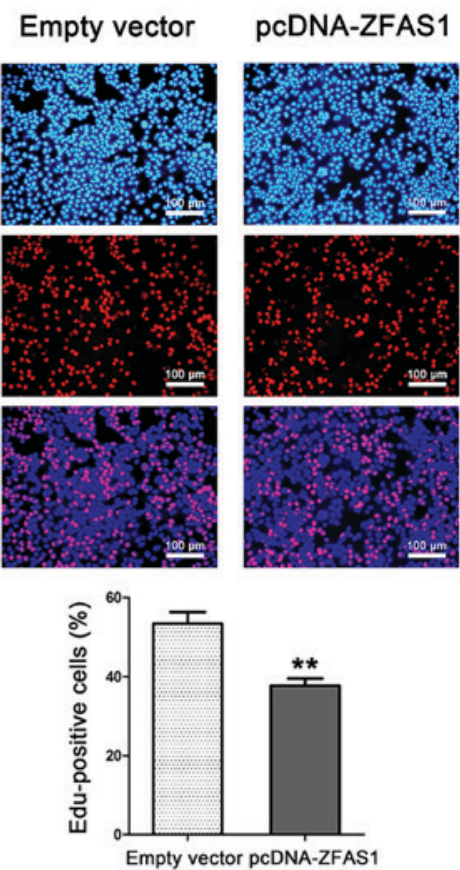

Figure 2. Effect of ZFAS1 upregulation on cell proliferation. Cell proliferation was detected by (A) Cell Counting Kit-8, (B) colony formation (scale bars, $10 \mathrm{~mm}$ ) and $(\mathrm{C})$ EdU assays (scale bars, $100 \mu \mathrm{m}$ ). Inhibition of cell proliferation was observed in ZFAS1-overexpressing breast cancer cells from MCF-7 and MDA-MB-231 cell lines. All experiments were performed in triplicate. Data are presented as the mean \pm standard deviation. "P<0.05 and ${ }^{* *} \mathrm{P}<0.01$ vs. empty vector (control). OD, optical density; ZFAS1, zinc finger antisense 1; Edu, 5-Ethynyl-2-deoxyuridine.

Fig. 3A, cell cycle progression in ZFAS1-overexpressing cells was significantly stalled at the G1/G0 phase compared to that in control cells. Furthermore, cell apoptosis analysis by flow cytometry demonstrated that ZFAS1 overexpression markedly increased the percentage of apoptotic cells in both the MCF-7 and MDA-MB-231 cell lines compared to the percentage in control groups (Fig. 3B).

Overexpression of ZFASI inhibits breast cancer cell migration and invasion by regulating EMT. To explore the potential role of ZFAS1 in breast cancer metastasis, and investigate the effect of ZFAS1 on migration and invasion capacity, we performed a Transwell assay using MDA-MB-231 cells. MDA-MB-231 cells were transfected with pCDNA-ZFAS1 or empty plasmid (negative control). As shown in Fig. 4A, ZFAS1 overexpression markedly suppressed cell migratory and invasion capacity compared to that observed in the control group. As the EMT process is a vital mechanism for cell metastasis, we next examined the effect of ZFAS1 overexpression on EMT in breast cancer cells. Western blotting was performed to examine the expression of EMT-related markers in MDA-MB-231 cells. As expected, ZFAS1 overexpression increased the expression of 
A Empty vector

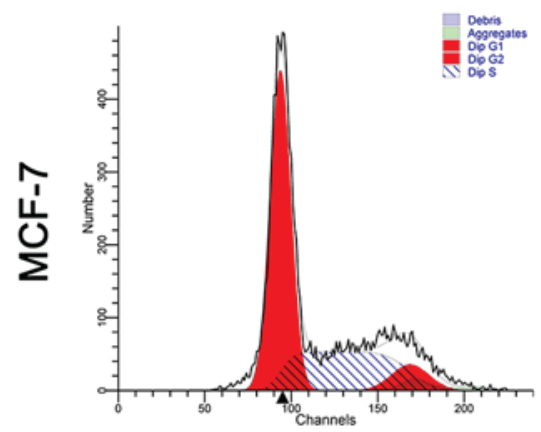

Empty vector

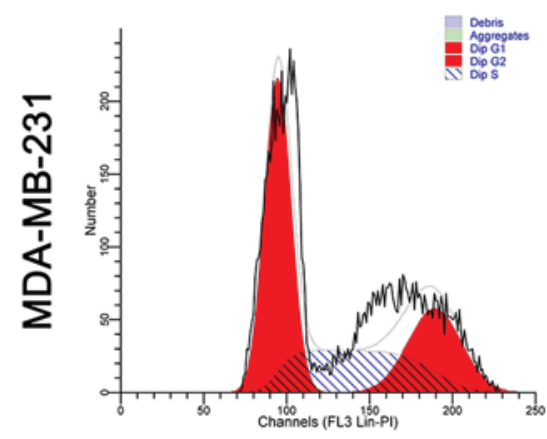

B

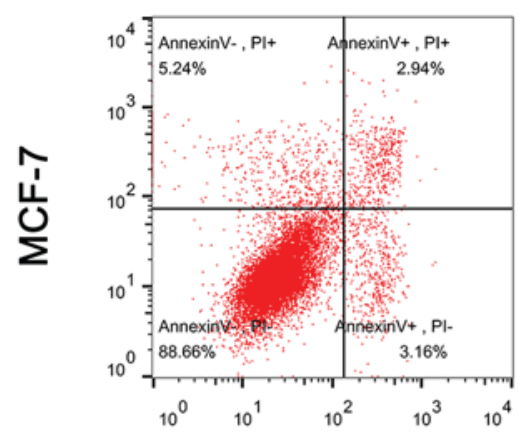

Empty vector

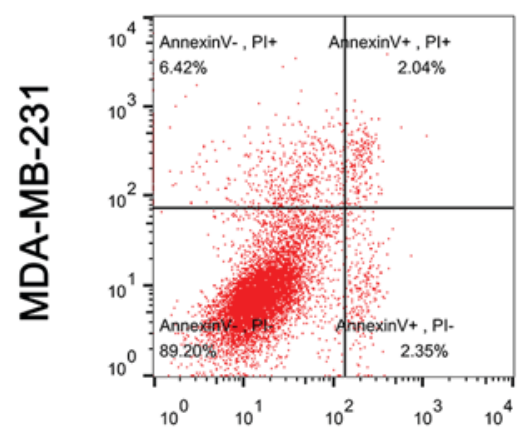

pcDNA-ZFAS1

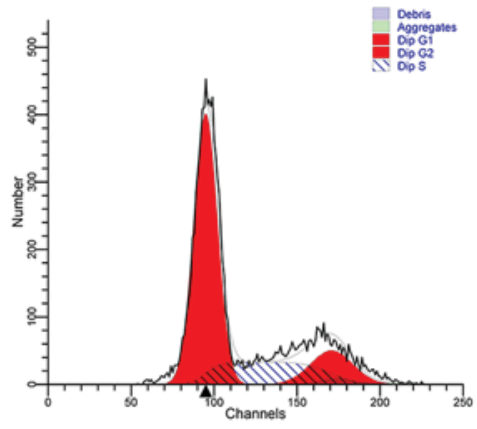

pcDNA-ZFAS1

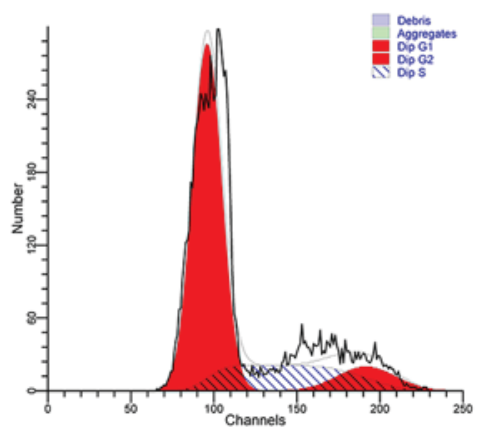

pcDNA-ZFAS1
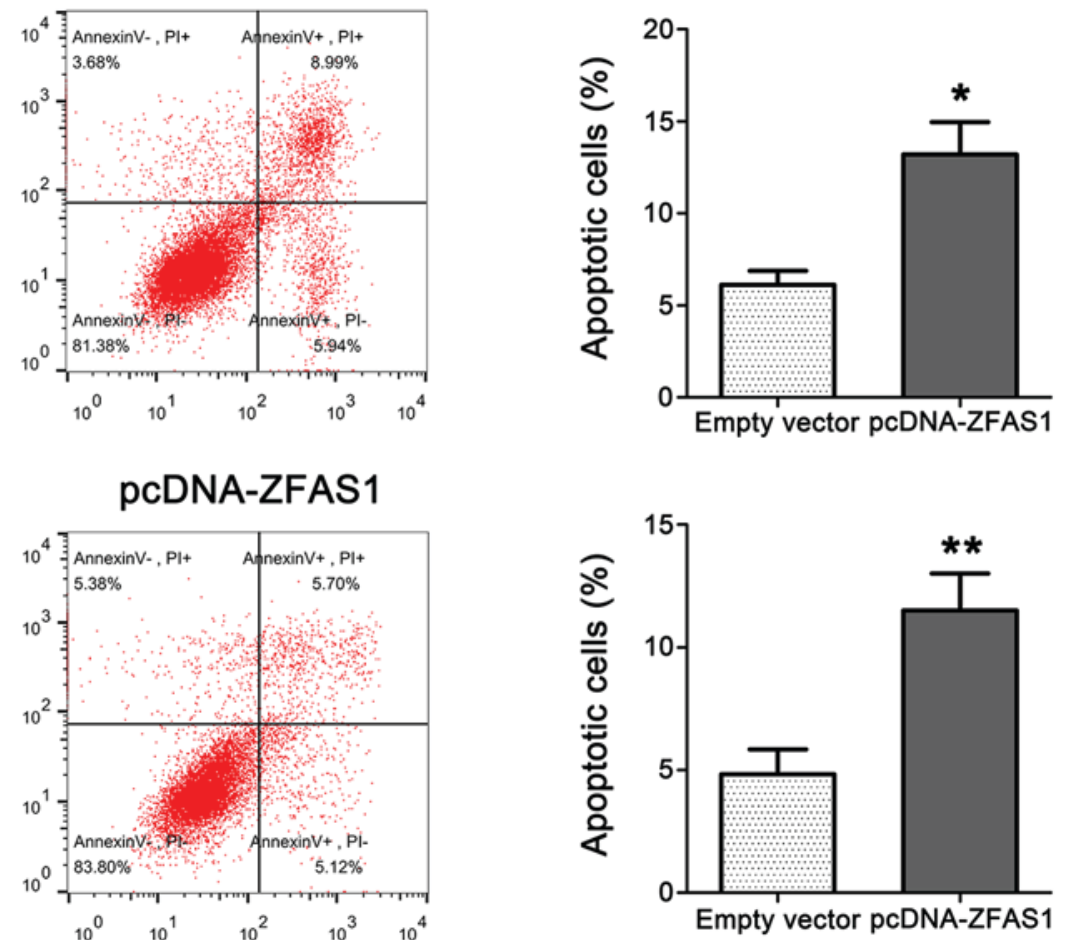

Figure 3. Effect of ZFAS1 upregulation on cell cycle and apoptosis. Cell cycle and cell apoptosis assays were conducted by flow cytometry. (A) Cell cycle arrest in the G1/G0 phase was observed in ZFAS1-overexpressing breast cancer cells from MCF-7 and MDA-MB-231 cell lines. (B) Cell apoptosis was induced by overexpressing ZFAS1 in breast cancer cells from MCF-7 and MDA-MB-231 cell lines. All experiments were performed in triplicate. Data are presented as the mean \pm standard deviation. ${ }^{*} \mathrm{P}<0.05$ and ${ }^{* *} \mathrm{P}<0.01$ vs. empty vector (control). ZFAS1, zinc finger antisense 1.

the epithelial marker E-cadherin while decreasing the expression of the mesenchymal markers N-cadherin and Vimentin in MDA-MB-231 cells (Fig. 4B).

\section{Discussion}

As the technologies for RNA profiling have improved over the 

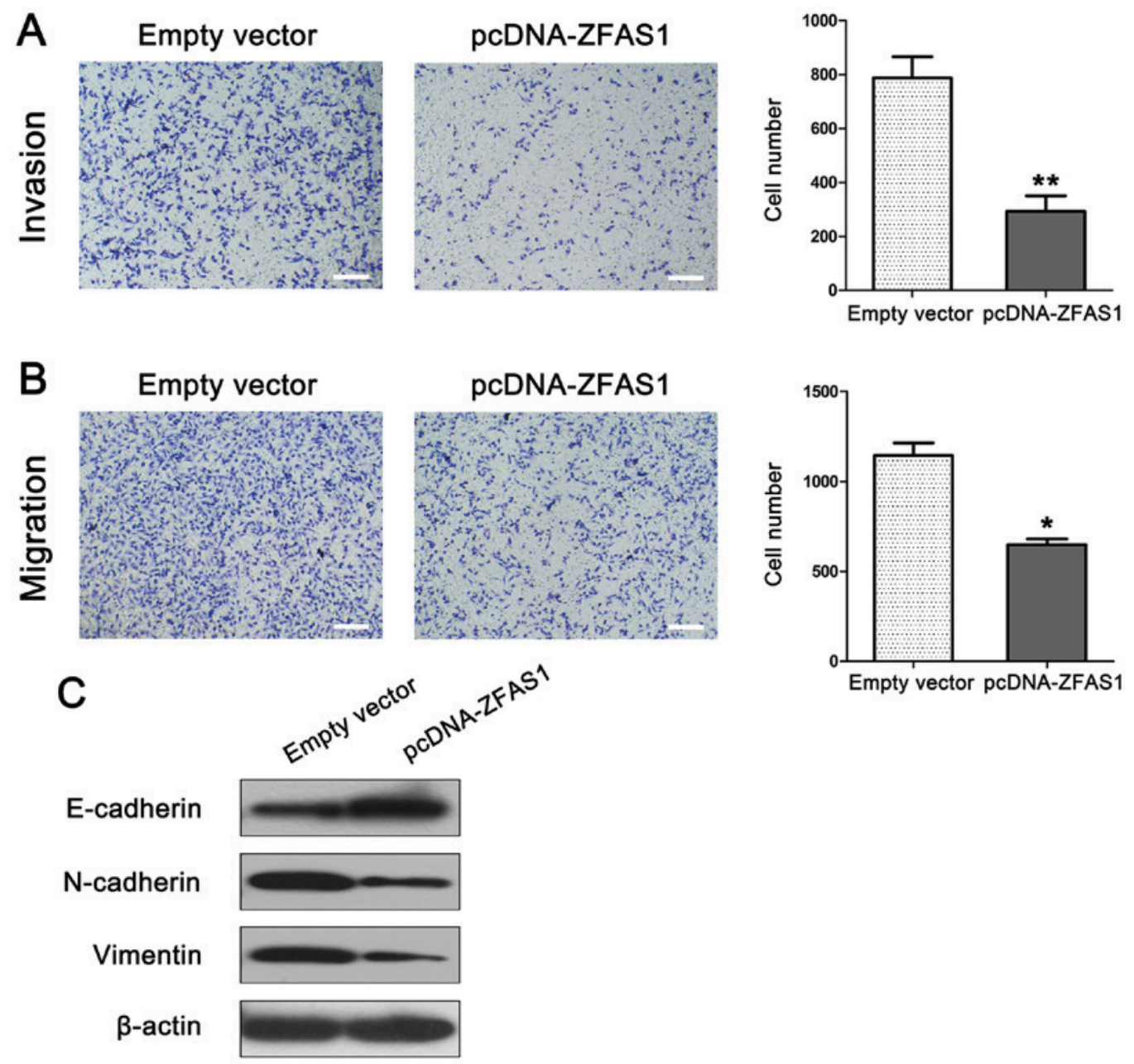

Figure 4. Effect of ZFAS1 upregulation on cell invasion, migration, and epithelial-mesenchymal transition. (A) Cell invasion and (B) migration were inhibited by overexpression of ZFAS1 in MDA-MB-231 breast cancer cells as determined by Transwell assays (scale bars, $100 \mu \mathrm{m}$ ). (C) ZFAS1 overexpression increased the expression of E-cadherin, and decreased the expression of N-cadherin and Vimentin in MDA-MB-231 cells as determined by western blotting. Data are presented as the mean \pm standard deviation of three independent experiments. Scale bars, $100 \mu \mathrm{m}$. ${ }^{*} \mathrm{P}<0.05$ and ${ }^{* *} \mathrm{P}<0.01$ vs. empty vector (control). ZFAS1, zinc finger antisense 1 .

past few decades, genome-wide transcriptome projects have detected large amounts of RNA that are transcribed but do not encode proteins. In fact, in humans only approximately $2 \%$ of the total genomic sequence is transcribed into protein-coding RNAs, with a vast proportion considered to be non-coding RNAs (ncRNAs) (15). The evolution and development of complex organisms require powerful and sophisticated regulatory systems, and the number of protein-coding genes alone cannot explain organismal complexity. Alternatively, ncRNAs, which account for a higher proportion of transcripts and exhibit complex sequences, may act as regulators of biological functions (16).

The advent of next-generation RNA sequencing technology, coupled with new computational methods for assembling transcriptomes, has led to the identification of a considerable number of lncRNAs. Transcript length (ranging from 200 to $>100,000 \mathrm{nt}$ ) and specific base pairing regions confer on lncRNAs the ability to fold into complex secondary structures. These secondary structures exert their characteristic functions by interacting with specific proteins and acting as scaffolds to form protein complexes (17). LncRNAs are a highly heterogeneous group of transcripts that regulate the expression of a wide variety of genes through diverse mechanisms including epigenetic, transcriptional and post-transcriptional regulation processes (18). In addition, IncRNAs have emerged as important regulators of gene expression in many cancers. For example, overexpression of the IncRNA LUCAT1 overexpression is associated with poor prognosis of non-small cell lung cancer and promotes cell proliferation through epigenetically regulating p21 and p57 expression (19). Similarly the lncRNA TUG1 promote gastric cancer cell proliferation by epigenetically silencing p57 expression (20). These findings indicate that IncRNAs may represent a novel family of tumor suppressor genes and oncogenes.

There is increasing evidence that aberrant expression of ZFAS1 plays a crucial role in carcinogenesis. A previous study revealed that ZFAS1 was highly expressed in gastric cancer promoting tumor growth and metastasis by inducing EMT (21). Another study showed that ZFAS1 may destabilize p53 and interacts with the CDK1/cyclin B1 complex, which leads to cell cycle progression and inhibition of apoptosis (22). Askarian-Amiri et al (10) found that ZFAS1 regulates epithelial cell proliferation and differentiation in the developing mouse mammary gland and that ZFAS1 is downregulated in 
human breast tumors compared to levels in normal tissues. However, few studies have investigated the biological roles of ZFAS1 in breast cancer.

To elucidate the role of ZFAS1 in the development of breast cancer, we measured ZFAS1 expression in cells from several breast cancer cell lines and a normal breast epithelial cell line, and performed a series of in vitro assays. We discovered that ZFAS1 expression in cells from breast cancer cell lines was significantly decreased compared to that found in MCF-10A cells and that increased expression of ZFAS1 inhibited breast cancer cell proliferation, migration, and invasion. Moreover, the dysregulation of cell cycle transitions is a hallmark of cancer cell (23). Thus, to investigate the possible mechanisms responsible for the effect of ZFAS1 on breast cancer cell proliferation, we used flow cytometry to determine that ZFAS1 overexpression inhibited cell proliferation by arresting the cell cycle at the G0/G1 phase and promoting cell apoptosis. These findings indicate that ZFAS1 may be a tumor suppressor in breast cancer and that the influence of ZFAS1 on cell proliferation may be associated with cell cycle and apoptosis control.

The migration and invasion of cancer cells are common events that mediate changes in cellular behavior, and lead to different steps in the metastatic process (24). EMT is a biological and pathological process whereby epithelial cells lose their apical-basal polarity and undergo a transition to a mesenchymal phenotype (25). It is considered to be a regulatory mechanism of metastasis in many cancers including breast cancer $(26,27)$. To further investigate the molecular mechanisms through which ZFAS1 inhibits the metastasis of breast cancer cells, we examined the expression of EMT-related markers in breast cancer cells with ZFAS1 overexpression. Our results showed that E-cadherin expression was upregulated while $\mathrm{N}$-cadherin and Vimentin expression were downregulated in ZFAS1-overexpressing cells, indicating that the effect of ZFAS1 on cell migration and invasion was partially associated with EMT process.

In conclusion, our findings from the current study demonstrate that ZFAS1 is downregulated in breast cancer cell lines. In addition, the overexpression of ZFAS1 inhibited cell proliferation, migration, invasion, and the EMT process. These results suggest that ZFAS1 is a tumor suppressor and potential therapeutic target for breast cancer, with further study required to more fully elucidate the underlying molecular mechanisms of ZFAS1 in breast cancer development.

\section{Acknowledgements}

Not applicable.

\section{Funding}

This study was supported by The National Natural Science Foundation of China (grant nos. 21131002, 21427802 and 21671076) and The Science and Technology Development Project of Jilin province (grant no. 20160101033JC).

\section{Availability of data and materials}

The datasets used and analyzed during the current study are available from the corresponding author on reasonable request.

\section{Authors' contributions}

SF carried out the experimental work, and data collection and interpretation. CF participated in the design and coordination of experimental work, and the acquisition of data. NL and $\mathrm{KH}$ participated in the study design, data collection, analysis of data and preparation of the manuscript. XF and KW carried out the study design, the analysis and interpretation of data, and drafted the manuscript. All authors read and approved the final manuscript.

\section{Ethics approval and consent to participate}

Not applicable.

\section{Consent for publication}

Not applicable.

\section{Competing interests}

The authors declare that they have no competing interests.

\section{References}

1. Torre LA, Bray F, Siegel RL, Ferlay J, Lortet-Tieulent J and Jemal A: Global cancer statistics, 2012. CA Cancer J Clin 65: 87-108, 2015.

2. Fatica A and Bozzoni I: Long non-coding RNAs: New players in cell differentiation and development. Nat Rev Genet 15: 7-21, 2014.

3. Ponting CP, Oliver PL and Reik W: Evolution and functions of long noncoding RNAs. Cell 136: 629-641, 2009.

4. Di Gesualdo F, Capaccioli S and Lulli M: A pathophysiological view of the long non-coding RNA world. Oncotarget 5: 10976-10996, 2014.

5. Wapinski $\mathrm{O}$ and Chang HY: Long noncoding RNAs and human disease. Trends Cell Biol 21: 354-361, 2011.

6. Yang L, Froberg JE and Lee JT: Long noncoding RNAs: Fresh perspectives into the RNA world. Trends Biochem Sci 39: 35-43, 2014.

7. Zhang EB, Yin DD, Sun M, Kong R, Liu XH, You LH, Han L, Xia R, Wang KM, Yang JS, et al: P53-regulated long non-coding RNA TUG1 affects cell proliferation in human non-small cell lung cancer, partly through epigenetically regulating HOXB7 expression. Cell Death Dis 5: e1243, 2014.

8. Zhou M, Zhang XY and Yu X: Overexpression of the long non-coding RNA SPRY4-IT1 promotes tumor cell proliferation and invasion by activating EZH2 in hepatocellular carcinoma. Biomed Pharmacother 85: 348-354, 2017.

9. Li J, Huang H, Li Y, Li L, Hou W and You Z: Decreased expression of long non-coding RNA GAS5 promotes cell proliferation, migration and invasion and indicates a poor prognosis in ovarian cancer. Oncol Rep 36: 3241-3250, 2016.

10. Askarian-Amiri ME, Crawford J, French JD, Smart CE, Smith MA, Clark MB, Ru K, Mercer TR, Thompson ER, Lakhani SR, et al: SNORD-host RNA Zfas1 is a regulator of mammary development and a potential marker for breast cancer. RNA 17: 878-891, 2011.

11. Nie F, Yu X, Huang M, Wang Y, Xie M, Ma H, Wang Z, De W and Sun M: Long noncoding RNA ZFAS1 promotes gastric cancer cells proliferation by epigenetically repressing KLF2 and NKD2 expression. Oncotarget 8: 38227-38238, 2016.

12. Fang C, Zan J, Yue B, Liu C, He C and Yan D: Long Noncoding RNA ZFAS1 promotes the progression of colonic cancer by modulating ZEB1 expression. J Gastroenterol Hepatol 32: 1204-1211, 2016

13. Gao K, Ji Z, She K, Yang Q and Shao L: Long non-coding RNA ZFAS1 is an unfavourable prognostic factor and promotes glioma cell progression by activation of the Notch signaling pathway. Biomed Pharmacother 87: 555-560, 2017. 
14. Xia B, Hou Y, Chen H, Yang S, Liu T, Lin M and Lou G: Long non-coding RNA ZFAS1 interacts with miR-150-5p to regulate Sp1 expression and ovarian cancer cell malignancy. Oncotarget 8: 19534-19546, 2017.

15. Djebali S, Davis CA, Merkel A, Dobin A, Lassmann T, Mortazavi A, Tanzer A, Lagarde J, Lin W, Schlesinger F, et al: Landscape of transcription in human cells. Nature 489: 101-108, 2012.

16. Mattick JS: RNA regulation: A new genetics? Nat Rev Genet 5: 316-323, 2004

17. Rinn JL and Chang HY: Genome regulation by long noncoding RNAs. Annu Rev Biochem 81: 145-166, 2012.

18. Bonasio R and Shiekhattar R: Regulation of transcription by long noncoding RNAs. Annu Rev Genet 48: 433-455, 2014.

19. Sun Y, Jin SD, Zhu Q, Han L, Feng J, Lu XY, Wang W, Wang F and Guo RH: Long non-coding RNA LUCAT1 is associated with poor prognosis in human non-small lung cancer and regulates cell proliferation via epigenetically repressing p21 and p57 expression. Oncotarget 8: 28297-28311, 2017.

20. Zhang E, He X, Yin D, Han L, Qiu M, Xu T, Xia R, Xu L, Yin R and De W: Increased expression of long noncoding RNA TUG1 predicts a poor prognosis of gastric cancer and regulates cell proliferation by epigenetically silencing of p57. Cell Death Dis 7: e2109, 2016.

21. Zhou H, Wang F, Chen H, Tan Q, Qiu S, Chen S, Jing W, Yu M, Liang C, Ye S and Tu J: Increased expression of long-noncoding RNA ZFAS1 is associated with epithelial-mesenchymal transition of gastric cancer. Aging (Albany NY) 8: 2023-2038, 2016.
22. Thorenoor N, Faltejskova-Vychytilova P, Hombach S, Mlcochova J, Kretz M, Svoboda $M$ and Slaby O: Long non-coding RNA ZFAS1 interacts with CDK1 and is involved in p53-dependent cell cycle control and apoptosis in colorectal cancer. Oncotarget 7: 622-637, 2016.

23. Hanahan D and Weinberg RA: Hallmarks of cancer: The next generation. Cell 144: 646-674, 2011.

24. Sahai E: Illuminating the metastatic process. Nat Rev Cancer 7 : 737-749, 2007.

25. Lamouille S, Xu J and Derynck R: Molecular mechanisms of epithelial-mesenchymal transition. Nat Rev Mol Cell Biol 15: 178-196, 2014.

26. Wu Y, Sarkissyan M and Vadgama JV: Epithelial-mesenchymal transition and breast cancer. J Clin Med 5, 2016.

27. Li W, Jia G, Qu Y, Du Q, Liu B and Liu B: Long non-coding RNA (LncRNA) HOXA11-AS promotes breast cancer invasion and metastasis by regulating epithelial-mesenchymal transition. Med Sci Monit 23: 3393-3403, 2017.

This work is licensed under a Creative Commons Attribution-NonCommercial-NoDerivatives 4.0 International (CC BY-NC-ND 4.0) License. 